C-A/AP/\#217

September 2005

\title{
AGS Injection with an Additional Kicker in the A10 Straight Section
}

\author{
C.J. Gardner
}

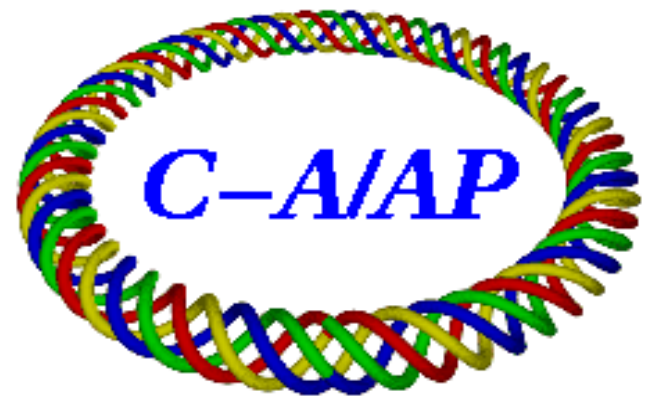

Collider-Accelerator Department Brookhaven National Laboratory Upton, NY 11973 


\title{
AGS Injection with an Additional Kicker in the A10 Straight Section
}

\author{
C.J. Gardner
}

September 13, 2005

Following are some notes on the possibility of AGS injection with an additional kicker in the A10 straight section.

Positive ions are injected into the AGS by means of a septum magnet (also called inflector) in the L20 straight and a kicker magnet in the A5 straight. We have known for some time that the A5 kicker does not provide enough kick for the optimum injection setup. As there is not enough room in the A5 straight for a stronger kicker, the possibility of putting an additional kicker in another straight section has been considered. The A20 straight is a desirable location because the phase advance from the exit of the septum magnet to the A20 straight is approximately $3 \pi / 2$. But this is also the optimum location for the superconducting helical dipole used to maintain the polarization of polarized protons. It was Woody Glenn who suggested that the A10 straight might be a viable alternative.

\section{Geometry and Parameters of the Injection Trajectory in the L20 Inflector}

The geometry and parameters of the injection trajectory in the L20 inflector are shown in Figure 1. Here incoming positive ions are indicated by the pink arrow. The trajectory is the circular arc shown in red. The radius of curvature of the arc is $\rho$ and the total turning angle is $\phi+\psi$. The length of the Beam Code axis is

$$
D_{1}+D+d+D_{2}=L_{3}=123.9907 \text { inches }
$$


as specified in $[1,2,3]$. The effective length of the inflector along the Beam Code axis is

$$
D+d=L_{1}+L_{2}+g
$$

where $g$ is the vertical gap in the septum magnet. According to AGS Department Drawing D37-M-0443 we have

$$
L_{1}=41.930 \text { inches }, \quad L_{2}=44.2337 \text { inches }, \quad g=1.840 \text { inches. }
$$

Thus

$$
D+d=88.0037 \text { inches }
$$

and therefore

$$
D_{1}+D_{2}=L 3-(D+d)=35.987 \text { inches. }
$$

We shall assume that the inflector is centered in the L20 straight section such that $D 1=D 2$. We then have

$$
D_{1}=D_{2}=17.9935 \text { inches. }
$$

According to Drawing D37-M-0426 we have

$$
\phi=6^{\circ} 38^{\prime} .
$$

The parameters $A+h$ and $\psi$ are the position and angle (with respect to the Beam Code axis) of the trajectory at the exit of the inflector. We define

$$
Y_{0}=A+h, \quad Y_{0}^{\prime}=\tan \psi
$$

and treat $Y_{0}$ as a free parameter. Once a value for $Y_{0}$ has been specified, the angle $\psi$ is determined by the requirement that the injection trajectory cross the equilibrium orbit at the A5 or A10 kicker. We then have radius of curvature

$$
\rho=\frac{D+d}{\sin \phi+\sin \psi}
$$

and

$$
\begin{gathered}
D=\rho \sin \phi, \quad d=\rho \sin \psi \\
L=\rho \tan (\phi / 2), \quad H=L \sin \phi \\
l=\rho \tan (\psi / 2), \quad h=l \sin \psi, \quad A=Y_{0}-h .
\end{gathered}
$$




\section{Position of Inflector Septum and Backleg}

The position of the inflector septum can be adjusted by rotating the entire inflector assembly about the pivot point shown in Drawing D37-M-0426.

In the fully inserted position the downstream tip of the septum conductor is 2.0541 inches from the Beam Code axis. Fully retracted the tip is 2.6165 inches from the Beam Code axis. These numbers come from Drawings D37-M-0426 and D37-M-0431. (Note that what I call fully retracted is labeled as "normal operating position" in drawing D37-M-0426.) What is not clear (to me) from the drawings is the orientation of the septum conductor in the fully inserted and retracted positions. If the fully retracted septum conductor is parallel to the Beam Code axis then the septum and backleg conductors are as shown in Figure 2. Here the brown, orange, and black lines show the conductors in the fully retracted, 0.25 inch inserted, and fully inserted positions respectively. The horizontal axis gives the distance $x$ along the Beam Code axis; the vertical axis gives minus the distance $y$ from the Beam Code axis. The units are inches.

If, on the other hand, the fully inserted septum conductor is parallel to the Beam Code axis then the septum and backleg conductors are as shown in Figure 3. Here again the brown, orange, and black lines show the conductors in the fully retracted, 0.25 inch inserted, and fully inserted positions respectively.

\section{Design Trajectory}

The original design trajectory was specified assuming that the septum was in the fully inserted position. The desired $Y_{0}$ and $Y_{0}^{\prime}$ of the trajectory at the exit of the inflector were found to be

$$
Y_{0}=3.2 \text { inches, } \quad Y_{0}^{\prime}=\tan \psi=4.5 \text { milliradians. }
$$

For these values equations (9-12) give

$$
\begin{gathered}
\rho=733.272 \text { inches } \\
D=84.704 \text { inches, } \quad d=3.300 \text { inches } \\
L=42.494 \text { inches, } \quad H=4.909 \text { inches } \\
l=1.650 \text { inches }, \quad h=0.0074 \text { inches }
\end{gathered}
$$


and

$$
A=3.193 \text { inches, } \quad A+H=8.101 \text { inches. }
$$

The trajectory is shown in red in Figures $\mathbf{4}$ and $\mathbf{5}$. Here, as before, the horizontal axis gives the distance $x$ along the Beam Code axis; the vertical axis gives minus the distance $y$ from this axis. The units are inches. The blue curves in the figures show the beam envelope assuming an emittance of $24 \pi \mathrm{mm}$ milliradians with the beam matched to Courant-Snyder parameters $\alpha=-1.355$ and $\beta=17.731$ meters at the inflector exit. The black lines show the septum conductor and backleg positions. In both figures the septum is fully inserted. In Figure 4 this is shown for the case in which the fully retracted septum is parallel to the Beam Code axis. Here we see that the beam envelope scrapes against the septum conductor. In Figure 5 the inserted septum is parallel to the Beam Code axis. Here the beam envelope fits cleanly between the septum and backleg. Note that $24 \pi \mathrm{mm}$ milliradians is the value originally given by Bleser for the acceptance of the L20 inflector.

\section{Some Algebra}

Let $\alpha$ and $\beta$ each with subscripts 0,1 , and 2 be the Courant-Snyder parameters at the L20 inflector exit, the A5 kicker, and the A10 kicker respectively. (We assume that the kicker magnets are point dipoles located at the centers of the A5 and A10 straights.) Then the elements of the transfer matrix from the inflector exit to the A5 kicker are

$$
\begin{gathered}
M_{11}=\sqrt{\beta_{1} / \beta_{0}}\left\{\cos \psi_{1}+\alpha_{0} \sin \psi_{1}\right\}, \quad M_{12}=\sqrt{\beta_{1} \beta_{0}} \sin \psi_{1} \\
M_{21}=-\left(\frac{\alpha_{1}-\alpha_{0}}{\sqrt{\beta_{1} \beta_{0}}}\right) \cos \psi_{1}-\left(\frac{1+\alpha_{1} \alpha_{0}}{\sqrt{\beta_{1} \beta_{0}}}\right) \sin \psi_{1} \\
M_{22}=\sqrt{\beta_{0} / \beta_{1}}\left\{\cos \psi_{1}-\alpha_{1} \sin \psi_{1}\right\}
\end{gathered}
$$

where $\psi_{1}$ is the betatron phase from the inflector exit to the kicker.

Similarly, the elements of the transfer matrix from the A5 kicker to the A10 kicker are

$$
\begin{gathered}
N_{11}=\sqrt{\beta_{2} / \beta_{1}}\left\{\cos \psi_{2}+\alpha_{1} \sin \psi_{2}\right\}, \quad N_{12}=\sqrt{\beta_{2} \beta_{1}} \sin \psi_{2} \\
N_{21}=-\left(\frac{\alpha_{2}-\alpha_{1}}{\sqrt{\beta_{2} \beta_{1}}}\right) \cos \psi_{2}-\left(\frac{1+\alpha_{2} \alpha_{1}}{\sqrt{\beta_{2} \beta_{1}}}\right) \sin \psi_{2}
\end{gathered}
$$


and

$$
N_{22}=\sqrt{\beta_{1} / \beta_{2}}\left\{\cos \psi_{2}-\alpha_{2} \sin \psi_{2}\right\}
$$

where $\psi_{2}$ is the betatron phase from the A5 to the A10 kicker. The elements of the transfer matrix from the inflector exit to the A10 kicker are

$$
\begin{gathered}
L_{11}=\sqrt{\beta_{2} / \beta_{0}}\left\{\cos \psi+\alpha_{0} \sin \psi\right\}, \quad L_{12}=\sqrt{\beta_{2} \beta_{0}} \sin \psi \\
L_{21}=-\left(\frac{\alpha_{2}-\alpha_{0}}{\sqrt{\beta_{2} \beta_{0}}}\right) \cos \psi-\left(\frac{1+\alpha_{2} \alpha_{0}}{\sqrt{\beta_{2} \beta_{0}}}\right) \sin \psi \\
L_{22}=\sqrt{\beta_{0} / \beta_{2}}\left\{\cos \psi-\alpha_{2} \sin \psi\right\}
\end{gathered}
$$

where

$$
\psi=\psi_{1}+\psi_{2}
$$

Now let

$$
\begin{gathered}
\mathbf{L}=\left(\begin{array}{ll}
L_{11} & L_{12} \\
L_{21} & L_{22}
\end{array}\right), \quad \mathbf{N}=\left(\begin{array}{ll}
N_{11} & N_{12} \\
N_{21} & N_{22}
\end{array}\right), \quad \mathbf{M}=\left(\begin{array}{cc}
M_{11} & M_{12} \\
M_{21} & M_{22}
\end{array}\right) \\
\mathbf{X}_{0}=\left(\begin{array}{c}
X_{0} \\
X_{0}^{\prime}
\end{array}\right), \quad \mathbf{X}_{1}=\left(\begin{array}{c}
X_{1} \\
X_{1}^{\prime}
\end{array}\right), \quad \mathbf{X}_{2}=\left(\begin{array}{c}
X_{2} \\
X_{2}^{\prime}
\end{array}\right)
\end{gathered}
$$

and

$$
\Phi_{1}=\left(\begin{array}{c}
0 \\
\phi_{1}
\end{array}\right), \quad \Phi_{2}=\left(\begin{array}{c}
0 \\
\phi_{2}
\end{array}\right), \quad \mathbf{0}=\left(\begin{array}{l}
0 \\
0
\end{array}\right) .
$$

Here $X_{0}$ and $X_{0}^{\prime}$ are the position and angle (with respect to the equilibrium orbit) of the incoming trajectory at the inflector exit; $X_{1}$ and $X_{1}^{\prime}$ are the position and angle (with respect to the equilibrium orbit) just after the A5 kicker; $X_{2}$ and $X_{2}^{\prime}$ are the position and angle (with respect to the equilibrium orbit) just after the A10 kicker; $\phi_{1}$ is the kick delivered by the A5 kicker; $\phi_{2}$ is the kick delivered by the A10 kicker. We then have

$$
\mathbf{L}=\mathbf{N M}, \quad \mathbf{X}_{1}=\mathbf{M} \mathbf{X}_{0}+\Phi_{1}, \quad \mathbf{X}_{2}=\mathbf{N X}_{1}+\Phi_{2} .
$$

We require that the position and angle (with respect to the equilibrium orbit) just after the A10 kicker be zero. Thus

$$
\mathbf{X}_{2}=\mathbf{0}
$$

and we have

$$
\mathbf{N X}_{1}+\Phi_{2}=\mathbf{0}
$$




$$
\begin{aligned}
\mathbf{N}\left(\mathbf{M X}_{0}+\Phi_{1}\right)+\Phi_{2} & =\mathbf{0} \\
\mathbf{M X}_{0}+\Phi_{1}+\mathbf{N}^{-1} \Phi_{2} & =\mathbf{0} \\
\mathbf{X}_{0}+\mathbf{M}^{-1} \Phi_{1}+\mathbf{L}^{-1} \Phi_{2} & =\mathbf{0}
\end{aligned}
$$

Thus

$$
\mathbf{X}_{0}=-\mathbf{M}^{-1} \Phi_{1}-\mathbf{L}^{-1} \Phi_{2}
$$

where

$$
\mathbf{M}^{-1}=\left(\begin{array}{rr}
M_{22} & -M_{12} \\
-M_{21} & M_{11}
\end{array}\right), \quad \mathbf{L}^{-1}=\left(\begin{array}{rr}
L_{22} & -L_{12} \\
-L_{21} & L_{11}
\end{array}\right)
$$

The components of $\mathbf{X}_{0}$ are then

$$
X_{0}=M_{12} \phi_{1}+L_{12} \phi_{2}, \quad X_{0}^{\prime}=-M_{11} \phi_{1}-L_{11} \phi_{2} .
$$

This is the key result. For given kick angles $\phi_{1}$ and $\phi_{2}$, the first equation tells us how much separation we have between the incoming trajectory and the equilibrium orbit at the inflector exit. (This separation has to accommodate the beam width and the septum thickness.) The second equation tells us what angle is needed between the incoming trajectory and the equilibrium orbit. Note that for the case in which $\phi_{2}=0$ (no kick at A10) we have

$$
X_{0}=M_{12} \phi_{1}, \quad X_{0}^{\prime}=-M_{11} \phi_{1}=-\left(M_{11} / M_{12}\right) X_{0}
$$

which is the familiar result for a single kick. Likewise for the case in which $\phi_{1}=0$ (no kick at A5) we have

$$
X_{0}=L_{12} \phi_{2}, \quad X_{0}^{\prime}=-L_{11} \phi_{2}=-\left(L_{11} / L_{12}\right) X_{0} .
$$

\section{Some Numbers}

With horizontal and vertical tunes set to $Q_{H}=8.80$ and $Q_{V}=8.75$, the MAD program gives

$$
\begin{gathered}
\alpha_{0}=-1.450, \quad \beta_{0}=17.68 \text { meters } \\
\beta_{1}=23.84 \text { meters, } \quad \beta_{2}=15.71 \text { meters } \\
\psi_{1}=58.6^{\circ}, \quad \psi_{2}=58.5^{\circ}, \quad \psi=\psi_{1}+\psi_{2}=117.1^{\circ} .
\end{gathered}
$$


Equations (19) and (25) then give

$$
M_{12}=17.53 \text { meters }, \quad M_{11}=-0.8340
$$

and

$$
L_{12}=14.83 \text { meters, } \quad L_{11}=-1.647 .
$$

With these values we see that the contribution of the A10 kick to $X_{0}$ in the first of equations (40) is just $85 \%$ of the contribution of the A5 kick. Note also that because $X_{0}, M_{12}$, and $L_{12}$ are all positive, optimum use of the two kicks requires that $\phi_{1}$ and $\phi_{2}$ both be positive.

In the second of equations (40) we see that the contribution of $\phi_{2}$ to $X_{0}^{\prime}$ is about twice that of $\phi_{1}$. This means that the angle (with respect to the equilibrium orbit) of the incoming trajectory at the inflector exit has to be increased by twice as much as it would have to be increased if the additional kick was coming from A5 rather than A10.

\section{Injection with No A10 Kick. No Orbit Bumps}

Suppose now that there is no kick at A10. The kick required at A5 is then

$$
\phi_{1}=X_{0} / M_{12}
$$

where $M_{12}$ is given by (46). With no orbit bumps, the equilibrium orbit in the L20 straight is parallel to and 0.13 inches to the inside of the Beam Code axis as shown in $[1,2,3]$. This means that for the design trajectory discussed in Section 3 we must have

$$
X_{0}=Y_{0}+b=3.33 \text { inches }
$$

where $b=0.13$ inches. This gives

$$
\phi_{1}=4.82 \text { milliradians }
$$

and

$$
X_{0}^{\prime}=-M_{11} \phi_{1}=4.02 \text { milliradians }
$$

\section{A5 Kicker Strength}

Now, the integrated strength of the A5 kicker is [4]

$$
B L=K I
$$


where

$$
K=1.8718 \times 10^{-5} \mathrm{Tm} / \mathrm{A}
$$

and $I$ is the current in Amps. The maximum available current is 1100 Amps. For a particle with magnetic rigidity $B \rho$ the angular kick $\phi_{1}$ delivered by the kicker is given by

$$
\sin \phi_{1}=B L /(B \rho)=K I /(B \rho) .
$$

The kick angles at maximum current for protons with various kinetic energies (and momenta $c p$ ) are given in Table 1. The values of $M_{12} \phi_{1}$ and $-M_{11} \phi_{1}$ are also given (units are mm and milliradians respectively).

Table 1: Maximum $\phi_{1}, M_{12} \phi_{1}$, and $-M_{11} \phi_{1}$ for various proton kinetic energies. The units of $M_{12} \phi_{1}$ and $-M_{11} \phi_{1}$ are mm and milliradians.

\begin{tabular}{|c|c|c|c|c|c|}
\hline $\mathrm{KE}(\mathrm{GeV})$ & $c p(\mathrm{GeV})$ & $B \rho(\mathrm{Tm})$ & $\phi_{1}(\mathrm{mr})$ & $M_{12} \phi_{1}$ & $-M_{11} \phi_{1}$ \\
\hline 1.50 & 2.25 & 7.5069 & 2.743 & 48.1 & 2.29 \\
1.60 & 2.36 & 7.8671 & 2.617 & 45.9 & 2.18 \\
1.70 & 2.47 & 8.2250 & 2.503 & 43.9 & 2.09 \\
1.80 & 2.57 & 8.5810 & 2.399 & 42.1 & 2.00 \\
1.90 & 2.68 & 8.9352 & 2.304 & 40.4 & 1.92 \\
1.94 & 2.72 & 9.0764 & 2.269 & 39.8 & 1.89 \\
2.00 & 2.78 & 9.2879 & 2.217 & 38.9 & 1.85 \\
\hline
\end{tabular}

Here we see that with no kick at A10 and with no orbit bumps, the A5 kicker provides only about half of what is needed according to equations (48-50).

\section{Injection with Kicks at both A5 and A10. No Orbit Bumps}

Suppose now that we have kicks at both A5 and A10. We fix $\phi_{1}$ at the maximum value possible for a given proton momentum. With no orbit bumps we have, as before,

$$
X_{0}=Y_{0}+b=3.33 \text { inches. }
$$


Putting this in the first of equations (40) along with the maximum values of $M_{12} \phi_{1}$ from Table 1 gives

$$
\phi_{2}=\left(X_{0}-M_{12} \phi_{1}\right) / L_{12}
$$

for the kick required at A10. The required angle (with respect to the equilibrium orbit) of the incoming trajectory at the inflector exit is then

$$
X_{0}^{\prime}=-M_{11} \phi_{1}-L_{11} \phi_{2} .
$$

The values of $\phi_{2}$ and $X_{0}^{\prime}$ computed in this way are listed in Table 2 .

Table 2: Required kick $\phi_{2}$ at A10 and injection angle (with respect to equilibrium orbit) $X_{0}^{\prime}$ for various proton kinetic energies with no orbit bumps. Here A5 kick $\phi_{1}$ is set to the maximum kick available at each kinetic energy.

\begin{tabular}{|c|c|c|c|c|}
\hline $\mathrm{KE}(\mathrm{GeV})$ & $B \rho(\mathrm{Tm})$ & $\phi_{1}(\mathrm{mr})$ & $\phi_{2}(\mathrm{mr})$ & $X_{0}^{\prime}(\mathrm{mr})$ \\
\hline 1.50 & 7.5069 & 2.743 & 2.46 & 6.34 \\
1.60 & 7.8671 & 2.617 & 2.61 & 6.48 \\
1.70 & 8.2250 & 2.503 & 2.74 & 6.60 \\
1.80 & 8.5810 & 2.399 & 2.86 & 6.71 \\
1.90 & 8.9352 & 2.304 & 2.98 & 6.83 \\
1.94 & 9.0764 & 2.269 & 3.02 & 6.86 \\
2.00 & 9.2879 & 2.217 & 3.08 & 6.92 \\
\hline
\end{tabular}

Here we see that for protons with kinetic energies greater than $1.6 \mathrm{GeV}$, the required kick at A10 is greater than the maximum available A5 kick. So without orbit bumps the kicker at A10 would have to be stronger than the present kicker at A5.

\section{Injection Bumps}

The first of equations (40) shows that the required kicks $\phi_{1}$ and $\phi_{2}$ can be reduced by reducing $X_{0}$. This in turn will reduce $X_{0}^{\prime}$. Since $X_{0}$ and $X_{0}^{\prime}$ are the position and angle of the incoming trajectory with respect to the equilibrium orbit, their values can be reduced either by changing the position $Y_{0}$ and angle $Y_{0}^{\prime}$ of the incoming trajectory with respect to the Beam Code axis, or by changing the position and angle of the equilibrium orbit at the inflector exit. Now, the position $Y_{0}$ is restricted by 
the location of the inflector septum. If the value of $Y_{0}$ is too small, incoming beam will scrape on the septum and be lost as can be seen in Figure 4. The angle $Y_{0}^{\prime}$ is also restricted. If the value of $Y_{0}^{\prime}$ is too large then the incoming trajectory will scrape on the outer wall of the vacuum chamber in the magnets between L20 and A5. It also may not be in the good field region of these magnets. Thus in order to reduce the values of $X_{0}$ and $X_{0}^{\prime}$ we need to be able to manipulate the position and angle of the equilibrium orbit at the inflector exit. This is done with two orbit bumps called the L20 position and angle bumps. These are documented in [5].

The position bump is a $3 / 2$ lambda bump. It is used to adjust the horizontal position of the equilibrium orbit at the inflector exit. By moving the orbit closer to inflector septum, we reduce $X_{0}$. The price for this is a reduction in the available aperture for the circulating beam at the inflector and at the outer lobes of the bump.

The angle bump is a lambda bump. It is used to adjust the angle of the equilibrium orbit at the inflector exit. If the angle of the incoming beam with respect to the Beam Code axis is held fixed, then adjusting this bump allows one to get the value of $X_{0}^{\prime}$ required by the second of equations (40). The price for this is again a reduction in the available aperture for circulating beam at the lobes of the bump.

Note that excitation of the position bump alone will change the equilibrium orbit angle at the inflector exit. One can compensate for this by adjusting the angle bump.

The features of the L20 position and angle bumps are illustrated in

Table 3. Here the two bumps have been excited to produce an equilibrium orbit with position $25.4 \mathrm{~mm}$ and various angles at the inflector exit. The corresponding orbit positions and angles at the A5 and A10 kickers are also given. (The kickers are assumed to be point dipoles at the centers of the A5 and A10 straights.) The orbits were obtained from the MAD code with the horizontal and vertical tunes set to $Q_{H}=8.80$ and $Q_{V}=8.75$. The first row of numbers in the table was obtained by exciting only the position bump. Here we see that a substantial orbit angle (1.826 $\mathrm{mr}$ ) is produced at the inflector exit. In the second, third, and fourth rows the angle bump has been adjusted to reduce this angle. The price for this is an increase in orbit distortion at both A5 and A10.

The black curves in Figures 6, 7, 8, and $\mathbf{9}$ show the equilibrium orbits corresponding to the first, second, third, and fourth rows of numbers in Table 3 . The blue curves in the figures show the envelope of circulating 
Table 3: Positions and angles of equilibrium orbit at L20 inflector exit, center of A5 straight, and center of A10 straight. These are due to L20 position and angle bumps and have been obtained from MAD code. Units are $\mathrm{mm}$ and milliradians.

\begin{tabular}{|c|c|c|c|c|c|}
\hline \multicolumn{2}{|c|}{ L20 } & \multicolumn{2}{c|}{ A5 } & \multicolumn{2}{c|}{ A10 } \\
\hline Pos & Ang & Pos & Ang & Pos & Ang \\
\hline 25.4 & 1.826 & 11.08 & -1.137 & -9.177 & 0.091 \\
\hline 25.4 & 1.102 & -1.650 & -1.493 & -19.26 & 1.421 \\
\hline 25.4 & 0.505 & -12.14 & -1.783 & -27.46 & 2.517 \\
\hline 25.4 & 0.000 & -21.00 & -2.027 & -34.31 & 3.444 \\
\hline
\end{tabular}

beam with an emittance of $24 \pi \mathrm{mm}$ milliradians. The vertical violet lines mark the positions (from left to right) of the L20 inflector exit, the A5 kicker, and the A10 kicker. In Figures 7, 8, and 9 the maximum orbit distortion occurs in the L5, L6, L17, L18, A9, and A10 magnets.

Figures 10 and 11 are extended views of Figures 6 and 8 showing the bump residuals.

\section{Aperture at L20, A5, and A10}

Note that the positions and angles in Table 3 and in Figures 6 through 11 are given with respect to the unperturbed (i.e. with no orbit bumps) equilibrium orbit. As already noted, the unperturbed orbit in the L20 straight is parallel to and 0.13 inches to the inside of the Beam Code axis $[1,2,3]$. The same is true in the A10 straight. In the A5 straight the unperturbed orbit is parallel to and 0.19 inches to the inside of the Beam code axis. We assume that the kicker aperture is centered on the unperturbed orbit in the A5 and A10 straights.

With no orbit bump and with the downstream end of the septum at 2.0541 inches from the Beam Code axis, the available aperture for circulating beam at the inflector exit is $2.0541+0.13=2.1841$ inches $(55 \mathrm{~mm})$. With the L20 position and angle bumps adjusted to give an orbit position of 
$25.4 \mathrm{~mm}(1 \mathrm{inch})$ at the inflector exit, the available aperture becomes $2.1841-1=1.1841$ inches $(30 \mathrm{~mm})$. The half width of beam with an emittance of $24 \pi \mathrm{mm}$ milliradians is $21 \mathrm{~mm}$ at the inflector exit. This gives a gap of $9 \mathrm{~mm}$ between the septum and the circulating beam envelope at the inflector exit.

The full horizontal aperture of the A5 kicker is 5.0 inches as shown in Figure 1 of Ref. [4]. With the kicker aperture centered on the unperturbed equilibrium orbit in the A5 straight, we then have 5.0/2 $=2.5$ inches (64 $\mathrm{mm}$ ) of available aperture on either side of the orbit. Table 4 gives the radial (horizontal) extent of the circulating beam envelope at the center of the A5 and A10 straights for the various orbit bumps considered in Table 3. Here the first two columns give the orbit position and angle at the inflector exit. The labels "In" and "Out" refer to the radially inner and outer boundaries of the envelope. The emittance of the circulating beam is taken to be $24 \pi \mathrm{mm}$ milliradians as before. From the Table and from Figures 6 through 9, we see that the beam envelope in the A5 and A10 kickers stays within the available aperture of $\pm 64 \mathrm{~mm}$.

Table 4: Inner and outer boundaries of circulating beam envelope at center of A5 and A10 straights (units are $\mathrm{mm}$ ). The first two columns give the orbit position and angle at the inflector exit (units are $\mathrm{mm}$ and milliradians) The emittance of the circulating beam is taken to be $24 \pi \mathrm{mm}$ milliradians.

\begin{tabular}{|c|c|c|c|c|c|}
\hline \multicolumn{2}{|c|}{ Orbit at L20 } & \multicolumn{2}{c|}{ A5 Envelope } & \multicolumn{2}{c|}{ A10 Envelope } \\
\hline Pos & Ang & In & Out & In & Out \\
\hline 25.4 & 1.826 & -13.24 & 35.41 & -28.01 & 9.66 \\
\hline 25.4 & 1.102 & -25.89 & 22.59 & -37.85 & -0.67 \\
\hline 25.4 & 0.505 & -36.31 & 12.03 & -45.85 & -9.06 \\
\hline 25.4 & 0.000 & -45.12 & 3.12 & -52.55 & -16.08 \\
\hline
\end{tabular}




\section{Injection with Bumps and A5 Kick. No Kick at A10.}

Consider now injection with position and angle bumps and A5 kick, but no kick at A10. The position (with respect to the Beam Code axis) of the incoming trajectory at the inflector exit is

$$
Y_{0}=3.2 \text { inches }=81.28 \mathrm{~mm} \text {. }
$$

The angle is $Y_{0}^{\prime}$. Using the MAD code we track the incoming trajectory from the inflector exit to the A5 kicker. The value of $Y_{0}^{\prime}$ is adjusted so that the trajectory crosses the equilibrium orbit at the kicker. The kick $\phi_{1}$ at the kicker is then adjusted to put the incoming beam on the equilibrium orbit. The results are summarized in Table 5 .

Table 5: Injection with bumps and A5 kick $\phi_{1}$. No kick at A10.

\begin{tabular}{|c|c|c|c|c|}
\hline$Y_{0}$ & $Y_{0}^{\prime}$ & \multicolumn{2}{|c|}{ Orbit at L20 } & $\phi_{1}$ \\
\hline $\mathrm{mm}$ & $\mathrm{mr}$ & Pos & Ang & $\mathrm{mr}$ \\
\hline 81.28 & 3.883 & 0 & 0 & 4.797 \\
\hline 81.28 & 4.507 & 25.4 & 1.826 & 3.347 \\
\hline 81.28 & 3.790 & 25.4 & 1.102 & 3.351 \\
\hline 81.28 & 3.198 & 25.4 & 0.505 & 3.354 \\
\hline 81.28 & 2.698 & 25.4 & 0.000 & 3.357 \\
\hline
\end{tabular}

Here $Y_{0}^{\prime}$ and A5 kick $\phi_{1}$ are given for various settings of the position and angle bumps. The third and fourth columns of the table give the orbit position and angle at the inflector exit (units are $\mathrm{mm}$ and $\mathrm{mr}$ ).

The first row of the table gives the values of $Y_{0}^{\prime}$ and $\phi_{1}$ for the case of no bumps. Here we see that angle $Y_{0}^{\prime}=3.883$ milliradians is required at the inflector exit and a kick of 4.797 milliradians is required at A5. These values are in agreement with those obtained from equations (48-51).

The second row gives the values of $Y_{0}^{\prime}$ and $\phi_{1}$ for the case in which the position bump alone is excited to give an orbit position of $25.4 \mathrm{~mm}$ at the 
inflector exit. The orbit angle is $1.826 \mathrm{mr}$. The required trajectory angle $Y_{0}^{\prime}$ has increased to $4.5 \mathrm{mr}$. The kick required at A5 has decreased from 4.797 to $3.347 \mathrm{mr}$. This is the setup for the original design trajectory.

The third, fourth, and fifth rows give the values of $Y_{0}^{\prime}$ and $\phi_{1}$ for the case in which both bumps are excited to give orbit position $25.4 \mathrm{~mm}$ and angles $1.102,0.505$ and $0.0 \mathrm{mr}$ respectively at the inflector exit. Here we see that the required trajectory angle $Y_{0}^{\prime}$ decreases form 4.507 to $3.790,3.198$, and $2.698 \mathrm{mr}$. The kick required at A5 remains about the same (3.35 mr). The price for the reduction in $Y_{0}^{\prime}$ is a reduction in available aperture for circulating beam as seen in Figures 6 through 9.

Note that even for protons with just $1.5 \mathrm{GeV}$ kinetic energy, the A5 kicker provides a maximum kick of only $2.743 \mathrm{mr}$ (see Table 1 ), which is not enough to meet the requirements given in Table 5 . This means that we may be running with an orbit position of greater than $25.4 \mathrm{~mm}$ (1 inch) at the inflector exit. We also may be focusing the incoming beam so that its width is small (and not matched to the Courant-Snyder parameters of the AGS lattice) at the inflector exit. This would allow the incoming beam to be moved closer to the septum thereby reducing the kick required at A5.

\section{Injection with Bumps and with Kicks at Both A5 and A10}

Consider now injection with position and angle bumps and with kicks at both A5 and A10. The position (with respect to the Beam Code axis) of the incoming trajectory at the inflector exit is again

$$
Y_{0}=3.2 \text { inches }=81.28 \mathrm{~mm} \text {. }
$$

The angle is $Y_{0}^{\prime}$. The A5 kick is set to $\phi_{1}=2.217 \mathrm{mr}$, the maximum value possible for protons with $2 \mathrm{GeV}$ kinetic energy. Using the MAD code as before we track the incoming trajectory from the inflector exit to the A10 kicker. The value of $Y_{0}^{\prime}$ is adjusted so that the trajectory crosses the equilibrium orbit at the A10 kicker. The A10 kick $\phi_{2}$ is then adjusted to put the incoming beam on the equilibrium orbit. The results are summarized in Table 6.

Here $Y_{0}^{\prime}$ and A10 kick $\phi_{2}$ are given for various settings of the position and angle bumps. The third and fourth columns of the table give the orbit position and angle at the inflector exit (units are $\mathrm{mm}$ and $\mathrm{mr}$ ). 
Table 6: Injection with bumps and with kicks at both A5 and A10.

\begin{tabular}{|c|c|c|c|c|c|}
\hline$Y_{0}$ & $Y_{0}^{\prime}$ & \multicolumn{2}{|c|}{ Orbit at L20 } & $\mathrm{A} 5 \phi_{1}$ & $\mathrm{~A} 10 \phi_{2}$ \\
\hline $\mathrm{mm}$ & $\mathrm{mr}$ & $\mathrm{Pos}$ & $\mathrm{Ang}$ & $\mathrm{mr}$ & $\mathrm{mr}$ \\
\hline 81.28 & 6.638 & 0 & 0 & 2.217 & 2.959 \\
\hline 81.28 & 3.979 & 25.4 & 0.000 & 2.217 & 1.402 \\
\hline 81.28 & 4.464 & 25.4 & 0.505 & 2.217 & 1.382 \\
\hline 81.28 & 5.038 & 25.4 & 1.102 & 2.217 & 1.359 \\
\hline 81.28 & 5.734 & 25.4 & 1.826 & 2.217 & 1.332 \\
\hline
\end{tabular}

The first row of the table gives the values of $Y_{0}^{\prime}$ and $\phi_{2}$ for the case of no bumps. Here we see that angle $Y_{0}^{\prime}=6.638$ milliradians is required at the inflector exit and a kick of 2.959 milliradians is required at A10. These numbers are in agreement with those given in the last row of Table 2. The angle $Y_{0}^{\prime}=6.638 \mathrm{mr}$ results in a trajectory that is too far to the outside in the magnets between the inflector exit and the A5 kicker. In order to pass through the good field region of these magnets, $Y_{0}^{\prime}$ must be $4.5 \mathrm{mr}$ or less.

Rows 2 through 5 of the Table show that with the position and angle bumps adjusted to give an orbit position of $25.4 \mathrm{~mm}$ at the inflector exit, the orbit angle needs to be $0.505 \mathrm{mr}$ or smaller in order for $Y_{0}^{\prime}$ to be less than $4.5 \mathrm{mr}$. Since the orbit distortion is greater for the case of zero orbit angle (row 2), we take row 3 (with orbit angle $0.505 \mathrm{mr}$ ) to be the nominal setup for injection with kickers at both A5 and A10. The circulating beam envelope in this case is given in the third row of Table 4 and in Figure 8. The required kicks at A5 and A10 are 2.217 and $1.382 \mathrm{mr}$ respectively.

\section{Acknowledgement}

I would like to thank Leif Ahrens for his advice during the course of this work. In particular, it was Leif who stressed the importance of manipulating the equilibrium orbit at the inflector exit. 


\section{References}

[1] E.J. Bleser, "Where are the AGS Magnets", Accelerator Division Technical Note 215, May 20, 1985.

[2] E.J. Bleser, "The Optimum Central Orbit in the AGS", Accelerator Division Technical Note 217, July 22, 1985.

[3] C.J. Gardner, "Notes on Orbit Equations in the AGS", C-A/AP Note 164, September 2004.

[4] L.A. Ahrens and C.J. Gardner, "Determination of the AGS Injection Kicker Strength from Beam Measurements", C-A/AP Note 91, December 2002.

[5] C.J. Gardner, "Modeling Backleg Winding Bumps with the BEAM Program", Accelerator Division Technical Note 341, August 21, 1990. 


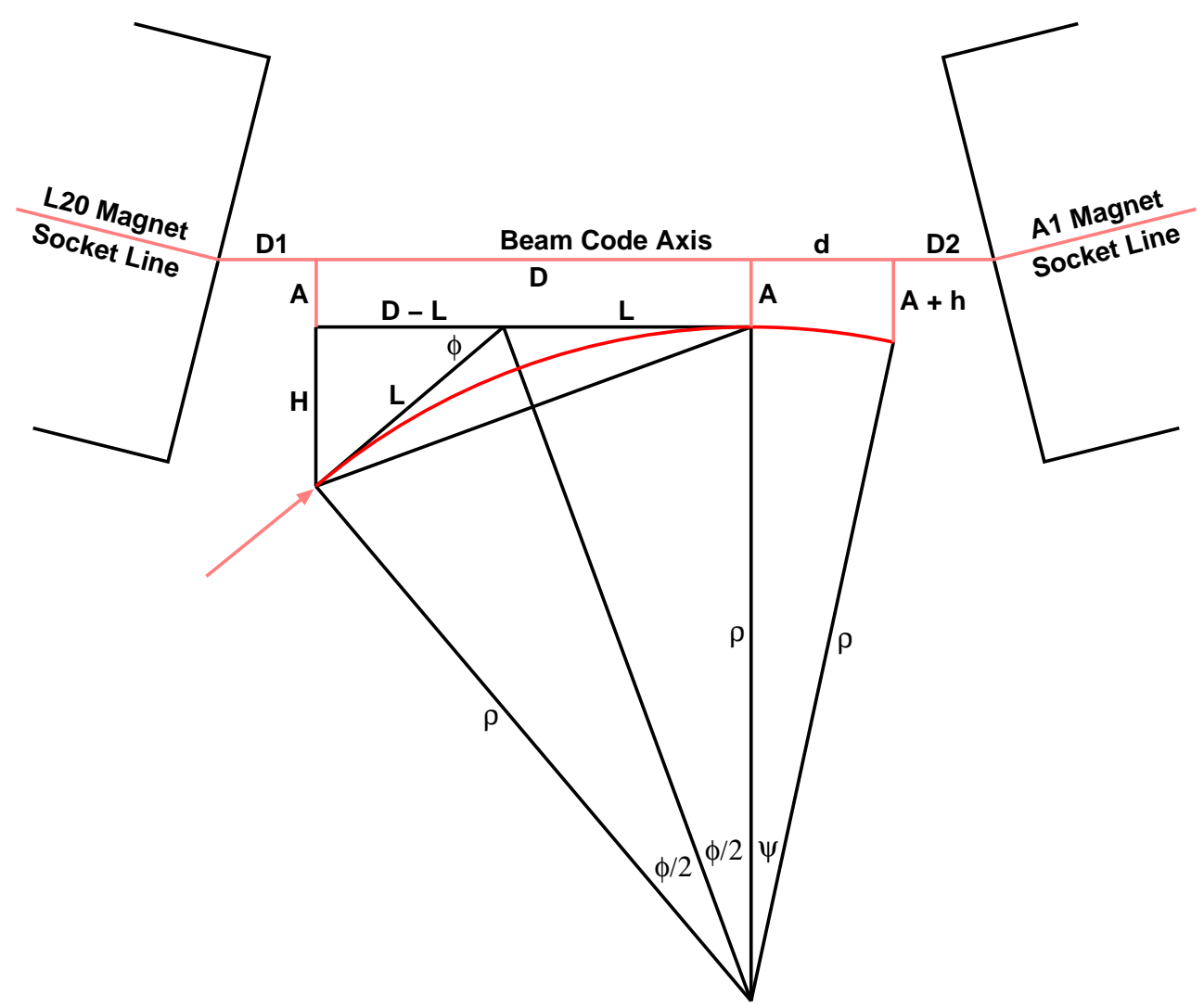

Figure 1: Geometry and parameters of the injection trajectory in the L20 inflector. Incoming positive ions are indicated by the pink arrow. The trajectory is the circular arc shown in red. The radius of curvature of the arc is $\rho$ and $\phi+\psi$ is the total turning angle. $A+h$ and $\psi$ are the position and angle of the trajectory with respect to the Beam Code axis at the inflector exit. The length of the Beam Code axis is $D_{1}+D+d+D_{2}$ and $D+d$ is the effective length of the inflector along this axis. 


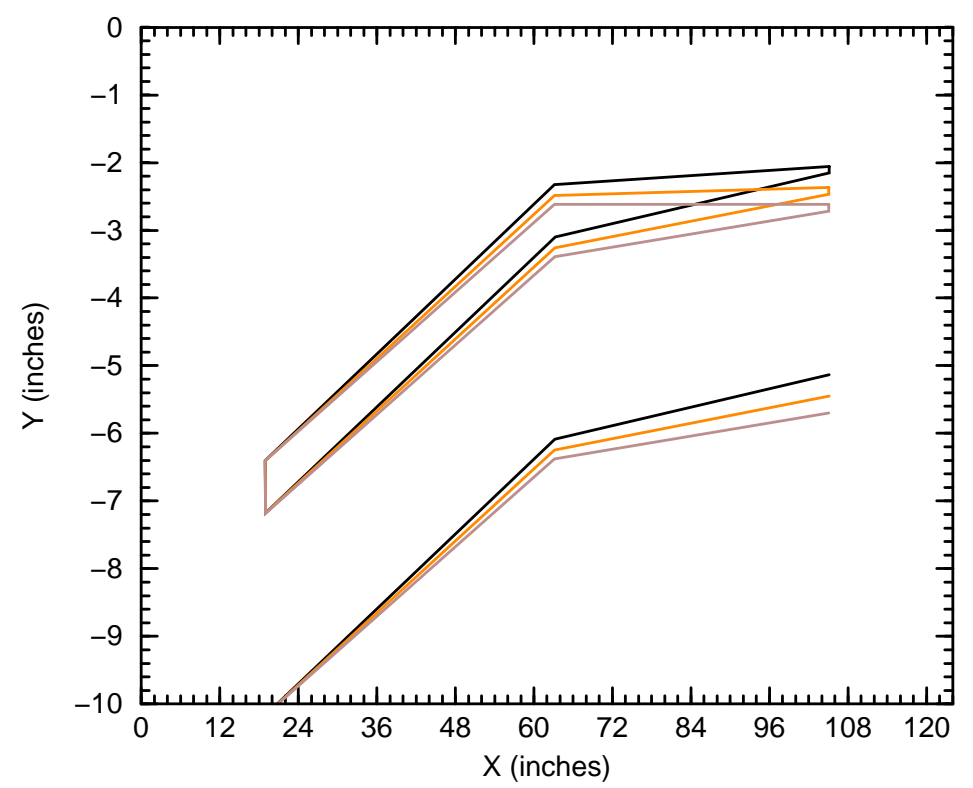

Figure 2: Septum and backleg positions for case in which the fully retracted septum is parallel to Beam Code axis.

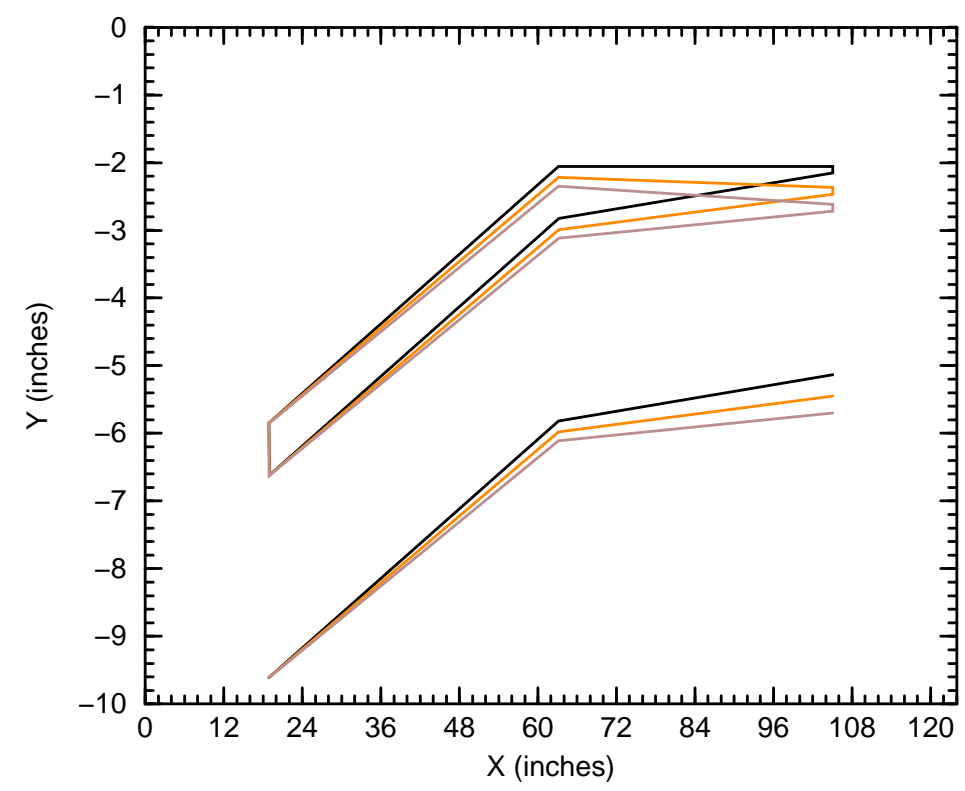

Figure 3: Septum and backleg positions for case in which the fully inserted septum is parallel to Beam Code axis. 


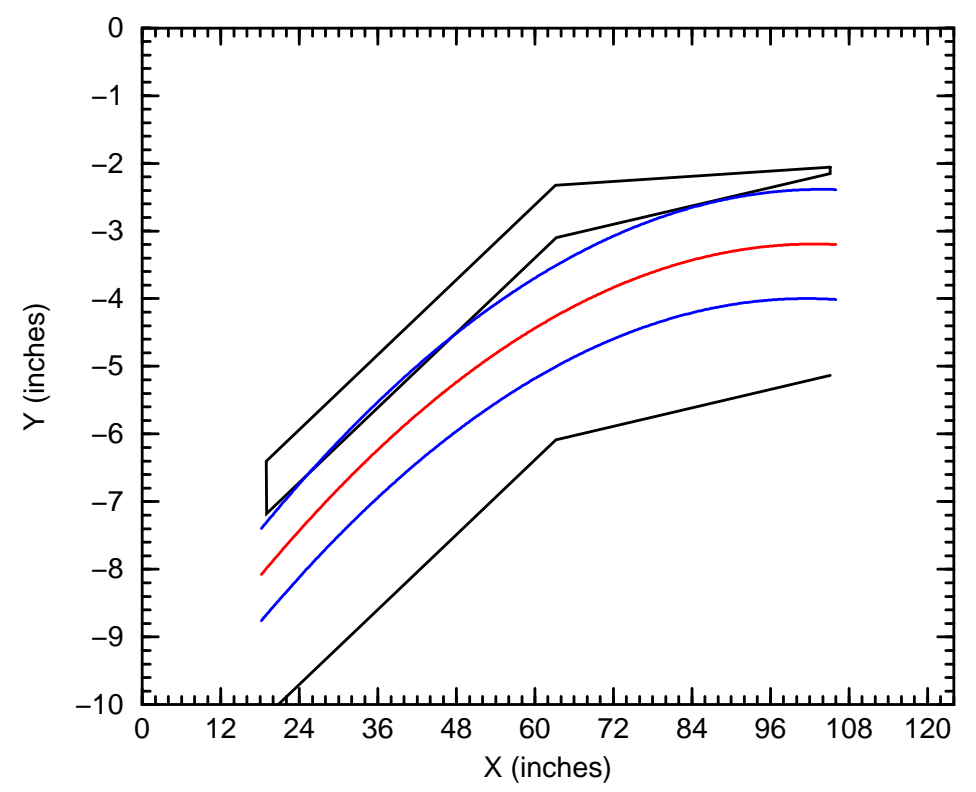

Figure 4: Original design trajectory with fully inserted septum for case in which fully retracted septum is parallel to Beam Code axis.

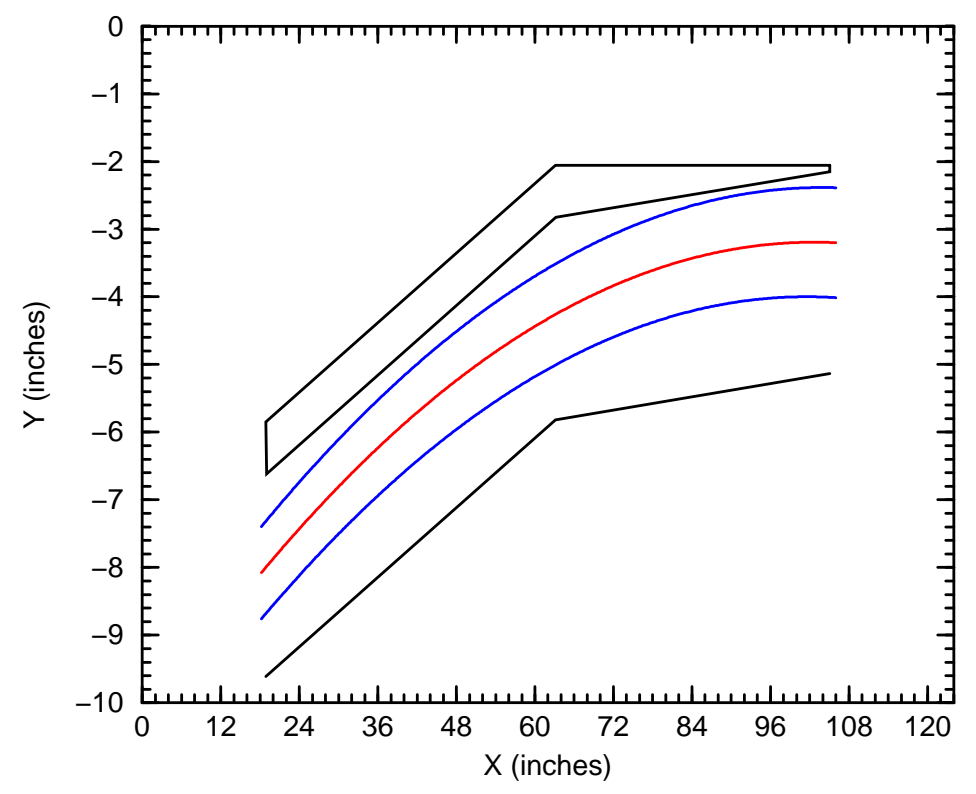

Figure 5: Original design trajectory with fully inserted septum parallel to Beam Code axis. 


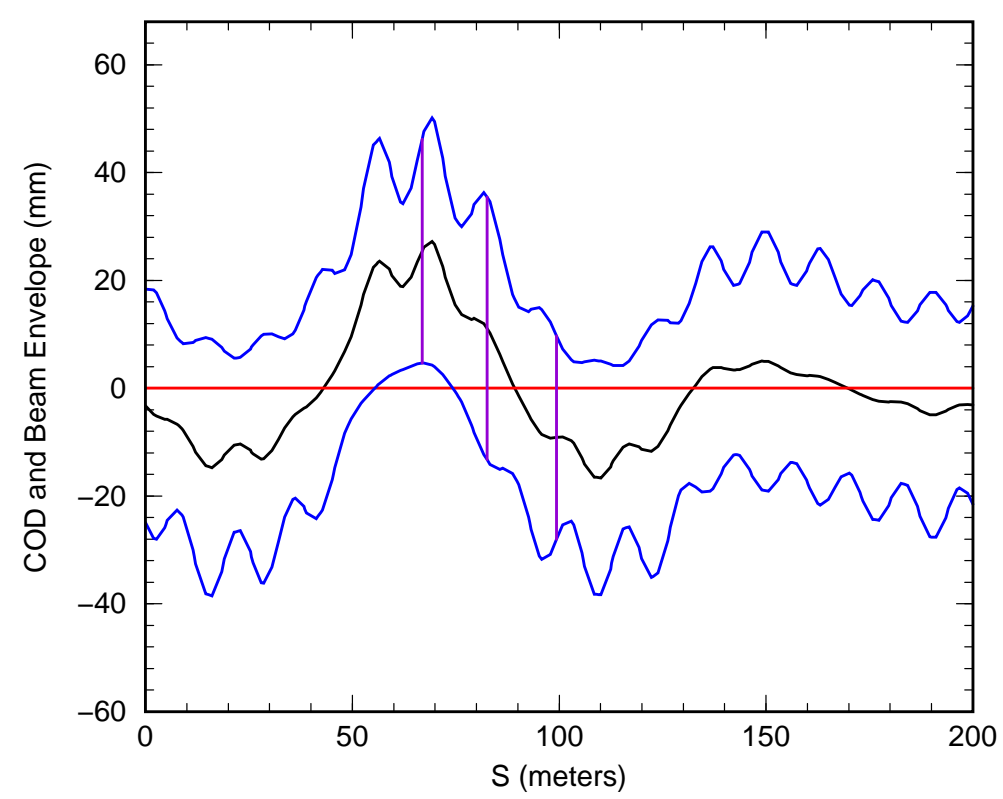

Figure 6: L20 position bump and beam envelope. Orbit position at inflector exit is $25.4 \mathrm{~mm}$; orbit angle is $1.826 \mathrm{mr}$.

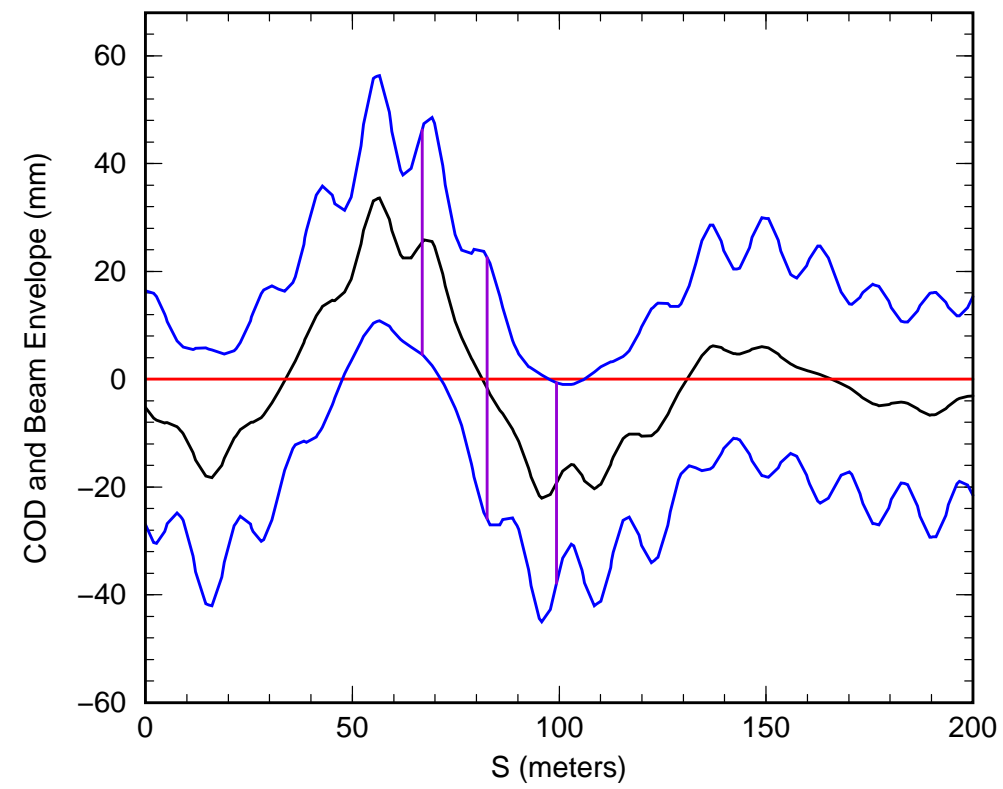

Figure 7: L20 position and angle bump and beam envelope. Orbit position at inflector exit is $25.4 \mathrm{~mm}$; orbit angle is $1.102 \mathrm{mr}$. 


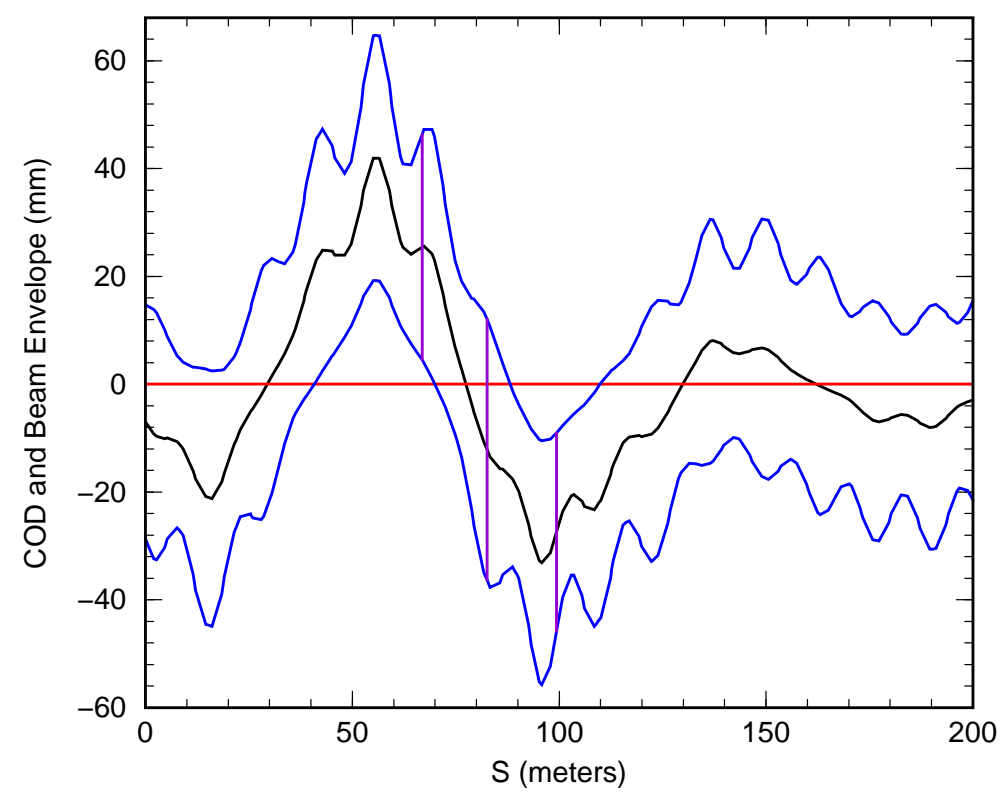

Figure 8: L20 position and angle bump and beam envelope. Orbit position at inflector exit is $25.4 \mathrm{~mm}$; orbit angle is $0.505 \mathrm{mr}$.

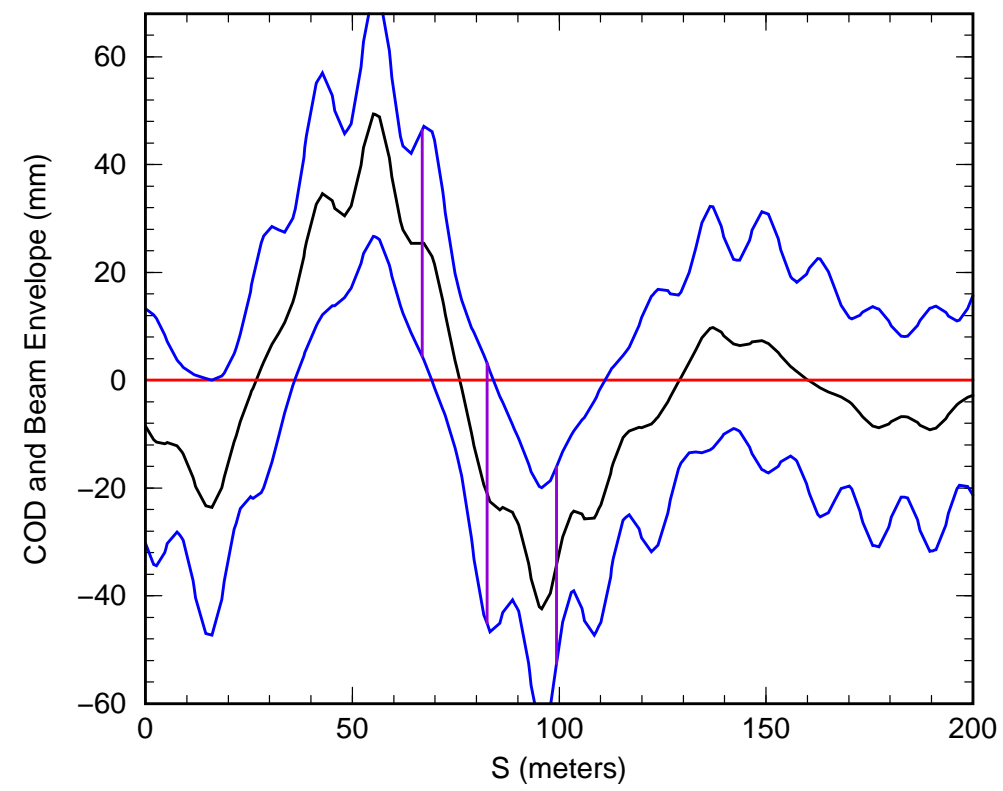

Figure 9: L20 position and angle bump and beam envelope. Orbit position at inflector exit is $25.4 \mathrm{~mm}$; orbit angle is $0.0 \mathrm{mr}$. 


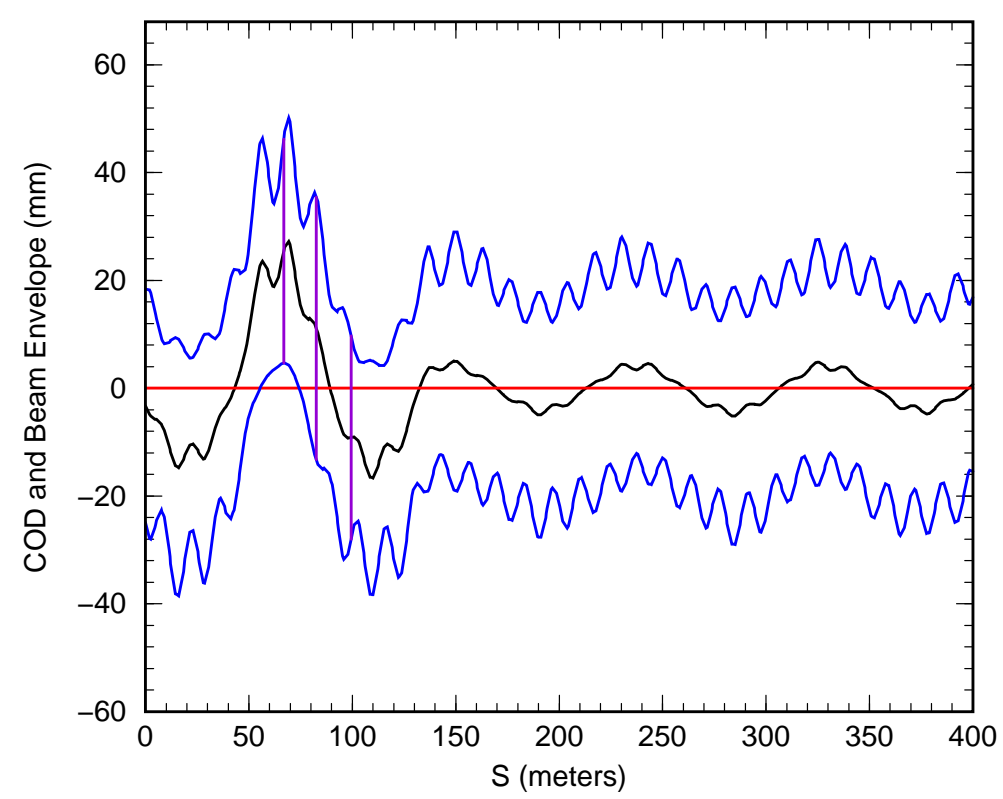

Figure 10: L20 position bump and beam envelope. Orbit position at inflector exit is $25.4 \mathrm{~mm}$; orbit angle is $1.826 \mathrm{mr}$.

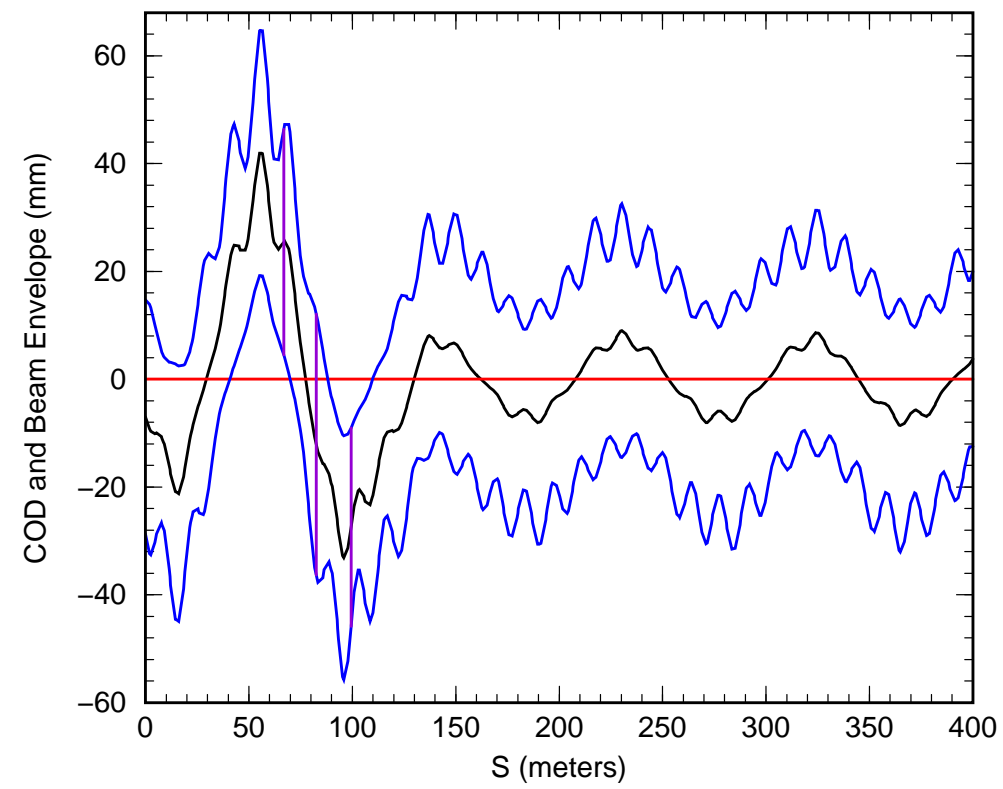

Figure 11: L20 position and angle bump and beam envelope. Orbit position at inflector exit is $25.4 \mathrm{~mm}$; orbit angle is $0.505 \mathrm{mr}$. 